\title{
ASPECTS OF “FIBROSITIS"
}

BY

\section{F. A. ELLIOTT}

The conception of fibrositis, introduced in its present form by Sir William Gowers in 1904, provided so ready an explanation for a number of otherwise knotty problems that it was and is widely accepted as a disease, although it has never been placed on a satisfactory pathological basis. It is true that Stockman, Brogsitter, and others have found non-inflammatory fibrotic changes and endarteritis in the subcutaneous and myo-fascial tissues, but the changes have been slight and-if we except the case of panniculitis in obese womentheir relation to symptoms indefinite. The scepticism born of this pathological ambiguity is increased by the circumstances that many conditions formerly viewed as typical examples of fibrositis are now known to have other cause. Many cases of lumbago, sciatica, and " brachial neuritis" (Semmes, et al., 1943; Spurling, et al., 1944; Elliott, et al.) are due to prolapse of the nucleus pulposus at the appropriate level. Other cases of lumbago are examples of spondylolisthesis, or the reverse condition of posterior displacement of the fifth lumbar vertebrae-conditions which depend for their recognition on improved $x$-ray technique. Orthopaedic surgery has demonstrated the importance of postural strains in the production of pain in the limb girdles and back. These advances have greatly reduced the number of cases in which it is necessary to invoke a hypothetical inflammation of fibrous tissues, and it is probable that this trend will continue. Nevertheless, it is still a common experience to find that cases of spondylitis, prolapsed disc, spinal tumour, and so on, have been treated as fibrositis by clinicians who are fully aware of these other diagnostic alternatives. It has appeared to me, from a review of personal mistakes of this kind, that among the factors which contribute to this confusion the most outstanding is the misinterpretation of tenderness as a physical sign. It is the purpose of this article to make some general observations on this topic, with special reference to muscle tenderness.

\section{Diagnostic Criteria for Fibrositis}

Modern literature emphasizes four points in the diagnosis of fibrositis: local tenderness, reproduction of symptoms by pressure on the tender points, the presence of nodules, and the cure of symptoms by the injection of procain into " trigger areas." A critical scrutiny of these criteria at once reveals certain pitfalls.
In the first place, it is not possible to map the distribution of tender spots by palpation alone, for it is a matter of simple physics that, since tenderness is elicited by pressure, it will be most easily found where there is something firm, like bone or ligament, to give counter-pressure to the exploring finger. Current descriptions of the common sites of "fibrositic" tenderness are for this reason unreliable, as can readily be demonstrated by exploring the tissues with a needle.

Secondly, tenderness found by palpation does not discriminate between superficial and deep tissues, so, unless special measures are adopted, the clinician is prone to attribute any tenderness he may find to the tissues he expects to find it in. Cutaneous hyperalgesia must be excluded by testing with pinprick. Subcutaneous tenderness must next be tested for by pinching up a roll of skin between the fingers, and is valid only if cutaneous hyperalgesia is absent. Tenderness in the deeper tissues is more difficult to judge and may require needling for its precise location. That these are not merely theoretical considerations may be illustrated by three cases, in all of which there was tenderness in the region of the sacro-iliac joint. One had an epidural abscess at the level of the third lumbar vertebra and the tenderness was of the skin, which here received fibres from the descending branch of the third lumbar nerve. The second had a prolapse of the fourth lumbar disc, irritating the fifth lumbar nerve, which supplies the gluteus maximus-the site of the tenderness. The third had tenderness deep to the myofascial strata, located by needling, and was due to a tuberculous lesion of the sacroiliac joint. All three had been regarded as cases of fibrositis because they had lumbago with a circumscribed area of local tenderness as described.

The third pitfall in the interpretation of tenderness is by far the most important, both practically and in its physiological implications. It is wrong to assume that local tenderness is necessarily due to local disease. In only one of the above examples did the tenderness indicate the site of the primary mischief. Tenderness means a reduction in the threshold for pressure pain. It implies an amplification of the response to pressure, and the process responsible for this amplification may be situated at any point in the sensory pathways, between the nerve endings in the tissue palpated and the thalamus. (For present purposes the tenderness which accompanies lesions in the spinal cord and thalamus will 
be excluded from the discussion.) Tenderness of the calf muscles may be due to local injury or local fatigue; but similar tenderness may be found when the responsible focus is an irritative lesion of the sciatic nerve, such as a gunshot wound in the thigh. It may be due to a root lesion, such as that caused by a prolapsed disc, or a tumour of the cauda equina. Local tenderness should not be accepted as proof of local disease unless there is satisfactory collateral evidence of a lesion in the area palpated. Even such evidence may be misleading, for it sometimes happens that some old-standing lesion such as a scar, or a joint injury, may become tender, and appear to be the source of pain by reason of its fortuitous presence within an area of referred tenderness from an irritative lesion of a nerve or nerve root. This fact, besides having interesting physiological implications, is clearly of practical importance and underlines the need for care in the elimination of central causes of tenderness. It can no longer be argued that the rarity of root syndromes reduces the importance of this observation. The advent of the prolapsed disc in clinical medicine has changed all that.

It is held by many that if the infiltration of procain into tender spots or nodules removes the patient's symptoms, the diagnosis of fibrositis is confirmed. This is not always true. I have pointed out (Elliott, 1944) that complete relief may be obtained by this measure when the tenderness is due to prolapsed discs and spinal tumours. Partial relief is more common in such cases, but whether partial or complete it is a paradoxical phenomenon. That it is susceptible of rational explanation will appear below. The practical issue is that a positive " novocain test" does not exclude a root lesion.

The fourth point stressed as diagnostic of fibrositis is the presence of nodules. But this, too, appears to offer practical difficulties, if we are to judge by the cases of spinal tumour and related conditions in which nodules have been found by competent observers in the early stages of the disease. The normal granularity of the subcutaneous tissue, and the coarse fascicular arrangement of some muscles are readily misinterpreted, particularly when they are tender. Biopsies have revealed many things: lipomas, neurofibromas, equivocal fibrosis, hyaline changes in muscle, and more often nothing at all. A striking instance of the last was a girl who had a tender nodule the size of a broad bean in the upper free edge of the trapezius. It was hard, and varied in size from day to day. She was given a general anaesthetic and the lump disappeared; an extensive search in the subcutaneous muscle, and submuscular layers revealed nothing amiss, and the lump never recurred. The virtue of this case lies in the fact that the nodule was visible as a hump under the skin, as well as being obvious to palpation. It appeared likely that the mass was due to spasm of muscle fibres in the trapezius, and it led to a series of observations on muscle tenderness which have produced new facts relevant to the problem of fibrositis. These will be outlined.

\section{Muscle Spasm and Tenderness}

In the first instance attention was confined to the tenderness of muscle occurring in proved root lesions. The site of the tenderness was established by differential testing. The traditional view of such hyperalgesia is that the impulses generated by palpation are normal impulses, but that they get amplified at some undetermined level in the central sensory pathways because they ascend via an irritated root. A second theory is that the presence of a root lesion increases the sensibility of the peripheral sensory endings in the tissue palpated. This controversy is unsettled and need not be discussed here. The outcome of the present investigations is to show that within these areas of increased sensory excitability, however they arise, there is in some cases a corresponding increase in motor irritability: that is to say, the tender muscles are more irritable than non-tender muscles, and are sometimes the seat of involuntary spasm involving small groups of muscle fibres. Such spasm produces both pain and tenderness, and becomes a source of pain over and above that which comes from the root lesion.

The possibility that subclinical spasm might account for some of the pain in these cases of root lesions was entertained on clinical grounds. It was submitted to instrumental investigation because it could not be either proved or disproved by palpation. In the electromyogram we have an instrument capable of accurate assessments of muscle activity. Electrodes are inserted into the muscle through a hypodermic needle, so bringing them into contact with muscle fibres at the tip of the needle. The electrodes are connected through a high-gain amplifier to a cathode-ray oscilloscope fitted with a camera. There is no electrical activity in muscle when it is at rest, but as soon as it contracts there is a change of potential which can be recorded on the oscilloscope.

The application of this method to the tender muscles in root lesions has been fully recorded elsewhere (Elliott, 1944). It was found that the tender muscles were irritable, responding to palpation and needling with prolonged outbursts of involuntary activity, denoted by showers of actionpotentials. At the most tender points there was in addition sustained motor activity which was not due to the presence of the needle, because in favourable cases it could be recorded from skin electrodes, and which lasted for several minutes at a time. It died away at once if procain was injected at the site of the discharge, and was abolished by a spinal anaesthetic. It occurred with the patient relaxed, and was obviously localized to a small portion of muscle, for by using a multi-channel recording system it was possible to take simultaneous records from tender and non-tender areas in the same muscle. Action currents came only from tender areas. Such particulate activity within a muscle which is otherwise at rest cannot be produced voluntarily and is thought to represent involuntary spasm. In radicular sciatica it has been recorded in tender "trigger" 
areas in the gluteus maximus and medius, and in the calf. In radicular pain in the upper extremity it has been found in the trapezius, supraspinatus, triceps, and extensors below the elbow. These are muscles with an extensor function, a distribution which has been constant thus far and which coincides, be it noted, with the usual sites of " rheumatic myalgia." It has not been found in purely flexor muscles.

The presence of these motor phenomena in the muscle innervated by irritated nerve roots supplies a possible explanation for the relief from pain which sometimes follows the injection of procain into tender areas within the territory of the affected root. It may be supposed that the root lesion gives rise to painful impulses which reflexly stimulate the motor cells of the anterior horns, so producing contraction of the corresponding groups of muscle fibres. Such contraction, if sustained, would give rise to both pain and tenderness, so increasing the flow of painful stimuli into the spinal cord and thereby exciting more spasm. If the reflex arc be cut, either by the injection of procain locally, or by a spinal anaesthetic, the spasm should stop and the relief from pain would be immediate. The degree of such relief would depend on how much of the patient's discomfort came from the root lesion and how much from the secondary spasm. If the former had become quiescent-as such lesions do-the spasm alone might be responsible for the pain, and in such a case the relief would be complete. Whether this hypothesis is correct or not, the abnormal irritability and sustained spasm demonstrated by the electromyogram introduce a new factor in the interpretation of muscle tenderness.

The findings in sciatica and "brachial neuritis" due to prolapsed discs encouraged an extension of this work to other forms of muscle tenderness. On theoretical grounds, any adequate stimulus coming in to the spinal cord should produce these motor changes, whether the source of the stimulus lies in a root or not. It appeared likely that an inflamed intervertebral joint, for instance, might give rise to spasm in the related segment of the erector spinae. In default of a suitably clear-cut case an artificial lesion was induced in a normal subject by injecting hypertonic saline into the sensitive area surrounding the articulation between the first and second lumbar vertebrae on one side. Unilateral subclinical spasm was induced in the sacrospinalis while the pain induced by the injection was at its height. This has been repeated in the dorsal area, but is more difficult owing to the difficulty which normal subjects have in relaxing the muscles of this region and the correspondingly high incidence of artefacts in the records obtained.

In two cases of long-standing thoracic scoliosis of undetermined aetiology pain and tenderness were referred to the erector spinae on one side at the level of the greatest deformity. The muscle here showed increased irritability and foci of spasm. Similar findings have been reported in an important paper by Hassett and Denslow (1942), who have studied postural strains in their relation to muscle fatigue. Failure to detect postural abnormalities will lead to an incorrect interpretation of local tenderness in such cases.

TWO CASES

Similar evidence of spasm has been encountered in two cases which correspond more closely to the traditional picture of fibrositis. The first was in a neurotic woman who developed acute pain and tenderness in the nuchal muscles, with occipital headache. She attributed it to sitting in a draught, but closer enquiry established that she had had a sudden severe "crick" in the neck while turning her head the day before. There was severe spasm in the nuchal group on one side, and a lesser degree on the opposite side. The head was held stiffly. Diagnosis here was a matter for speculation; but it was analogous to the lumbago of a prolapsed disc.

A second case was that of a woman, likewise highly strung, who suffered from periodical pain in the shoulders and neck. Two acute attacks followed the removal of septic teeth, and were accompanied by a temperature. There were numerous tender spots in the upper fibres of both trapezii, the levator scapulae, and the rhomboids. Pain was induced by movements of the neck which relaxed the affected muscles, and this pain was felt in the muscles and not in the neck. The tender muscles showed many areas of spasm. She was relieved by certain postures and by lying in a hot bath. It was difficult to avoid the conclusion that she had a localized arthritis of the cervical spine, with secondary spasm of the related muscles; but here again proof was lacking.

\section{Some Factors in Muscle Pain}

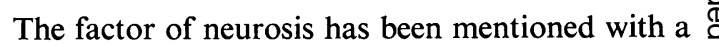
purpose. The incidence of muscle spasm has shown $\overrightarrow{\overrightarrow{0}}$ a direct relation to the severity of the local lesion and also to what may loosely be called the nervous constitution of the subject. It is more common in severe lesions than in slight, and in highly-strung subjects than in the phlegmatic. This is what would be expected if the presence of spasm depends, as postulated, on the establishment of a local reflex in the spinal cord. Facilitation from above will be greatest at moments of stress and worry-conditions which not only sharpen the perception of pain but also increase the reflex excitability of the nervous system. This is in accord with the known effect of such factors on " rheumatic" pains.

The part played by muscle pain varies from case to $N$ case. In many cases of prolapsed disc, for instance, స్心 there is no evidence of spasm, and the patient's dis- $O$ comfort comes from the local lesion, its effect on the posterior common ligament, and the nerve root. $\frac{\mathscr{C}}{\Phi}$ Even when spasm is present it makes a very variable $\stackrel{?}{?}$ contribution to the patient's total discomfort, as 0 can be judged from the unpredictable degree of relief from the injection of procain and other sub- $\stackrel{\mathbb{\Omega}}{\stackrel{\Omega}{\circ}}$ stances. In some cases the amount of pain which $\frac{}{\mathbb{1}}$ arises from relatively small foci of spasm is remark- $\frac{\varrho}{\sigma}$ able, and in searching for an explanation for this two things have become apparent. The first is that $\frac{8}{0}$ involuntary and " unphysiological " contraction of skeletal muscle is immediately painful, as in the흘 familiar case of cramps in the calf. This pain comes? 
on at once and is not dependent on the accumulation of the metabolic products to which muscle pain is attributed. It appears to be related to the distortion which is produced in a muscle when some of its fibres contract and others do not.

The second factor concerns the blood supply of the muscles. It is known that a reduction of the arterial supply to a muscle will bring about pain if that muscle is made to work, as in claudication. I have from time to time observed reduction and even temporary obliteration of the arterial pulsation at the ankle in the presence of severe sciatic pain; the foot has felt cold, but with rest and relief from pain the pulsation has returned to normal.

The clinically observed reduction in the blood supply to the limb has been confirmed by a thermoelectric study of muscle temperatures in subjects with root lesions. By using needle electrodes it is possible to record the temperature of the tissue in contact with the tip of the needle. This work is still proceeding, but it has been found that the tender muscles are " cooler" than those of the normal side, and that the distribution of this change shows that not all of it is due to disuse of the limb. The drop of temperature is especially marked in muscles which are the seat of spasm, and exceeds by far the limits of normal variation. Since the temperature of any tissue depends partly on its own metabolism and partly on the central-heating effect of its arterial blood supply, it follows that, if a muscle which is abnormally active is found to be cooler than inactive muscle in the opposite limb, the lowering of temperature must be due to reduction of blood supply. How this comes about need not be discussed here, but the fact that it occurs promises to be of importance in the study of muscle pain. In the present context the existence of a relative ischaemia supplies the conditions under which spasm of skeletal muscle is most likely to produce pain. Secondly, the existence of such ischaemia over long periods may prove a source of trophic changes in the tissues. The rapid wasting of muscles without a corresponding loss of power, which is so common in root lesions, may have a vascular basis. The rather equivocal fibrosis and endarteritis described by various authors as the fibrositic lesion may be the result of prolonged partial ischaemia. These studies are in an early stage of development and have not yet been extended to the wider field of non-radicular cases, but it appears probable that closer study of the vascular responses to pain in joints will prove worth while. They are mentioned here only in the most general way, pending further work on the subject. It is felt that, no less than electromyography, they offer a profitable line of attack on the problem of chronic rheumatism.

\section{Summary}

We have seen that there has been a progressive reduction in the number of conditions which can be attributed to fibrositis, and that the application of a rigorous clinical discipline coupled with new technical methods threatens to continue the process of dismemberment.

The present work seems to show that, apart from a hypothetical inflammatory origin, muscle tenderness may have two explanations. The first is the traditional one - a central lowering of threshold to sensory stimuli. The second is the effect of localized involuntary spasm arising reflexly from lesions elsewhere. This spasm is accompanied by an increased irritability and occurs in extensor muscles which are anatomically or physiologically related to the site of disease. Thus a prolapsed disc in the lumbar spine may give rise to spasm in the lumbar muscles - that is, an anatomically determined spasm-and it may also cause spasm of small groups of muscle fibres in the buttock and calf by irritating the fifth lumbar root. Such spasm contributes a variable and at times considerable amount of pain to the patient's discomfort, and this may be relieved by measures taken to reduce the spasm. Such treatment is valuable, but it has been pointed out that the elimination of pain by local injections of procain does not necessarily exclude a central lesion of the nerve roots or spinal column.

This thesis does not seek to shut the door on fibrositis as a clinical entity, and it would be unfortunate if the hypothesis of myofascial inflammation were to be replaced, at this stage, by a facile generalization that muscle tenderness is always due to spasm of skeletal muscle.

I have to thank Lieut.-Colonel Greenway, R.A.M.C., for his permission to publish this article.

\section{REFERENCES}

Denslow, J. S.. and Hassett, C. C. (1942). J. Neurophysiol., 5, 393. Elliott, F. A. (1944). Lancet, 1, 47

Elliott, F. A., and Kremer, M. (in Press)

Semmes, R. E., and Murphey, F. (1943). J. Amer. med. Ass., 121, 1209.

Spurling, R. G., and Scoville, W. B. (1944). Surg. Gynaecol. Obstet., 78, 350. 